Draft VERSION MARCH 1, 2022

Preprint typeset using $\mathrm{LATE}_{\mathrm{E}} \mathrm{X}$ style emulateapj v. 5/2/11

\title{
THE MASS OF FIRST STARS
}

\author{
Hajime Susa ${ }^{1}$ \\ Department of Physics, Konan University, Okamoto, Kobe, Japan \\ Draft version March 1, 2022
}

\begin{abstract}
We perform a three dimensional radiation hydrodynamics simulation to investigate the formation of first stars from initial collapse of a primordial gas cloud to formation and growth of protostars. The simulation is integrated until $\sim 0.1$ Myrs after the formation of the primary protostar by which the protostars have already settled onto main sequence stars. This is the first attempt of simulating first star formation to take into account the ultraviolet radiative feedback effect by the multiple protostars as well as the three dimensional effects such as fragmentation of the accretion disk. We find that the mass accretions onto the population III protostars are significantly suppressed by the radiative feedback from themselves. As a result, we find five stars formed in this particular simulation, and that the final mass of the stars are $\lesssim 60 M_{\odot}$, including a star of $4.4 M_{\odot}$. Formation of such a star hints at the existence of even lower-mass stars that would live today.
\end{abstract}

Subject headings: early universe - radiative transfer — first stars

\section{INTRODUCTION}

Formation of first stars has been investigated intensively in the last decade mainly from theoretical aspects. Following the theoretical predictions, the first stars form in the mini-halos of mass $\sim$ $10^{5}-10^{6} M_{\odot}$ (Haiman et al. 1996; Tegmark et al. 1997; Nishi \& Susa 1999; Fuller \& Couchman 2000; Abel et al. 2002; Bromm et al. 2002; Yoshida et al. 2003).

The ingredient of first star formation is the primordial gas, which does not contain heavy elements or cosmic dusts. Because of the lack of these efficient coolants, the primordial gas cools inefficiently especially at low temperatures $\left(T \lesssim 10^{4} \mathrm{~K}\right)$. Therefore, the gas is kept relatively warm $\left(\sim 10^{3} \mathrm{~K}\right)$ while it collapses to form stars, in contrast to the case of the interstellar gas, whose temperature is $\sim 10 \mathrm{~K}$ during the present-day star formation for $n_{\mathrm{H}} \lesssim 10^{10} \mathrm{~cm}^{-3}$ (e.g. Omukai 2000). As a result, the gravitationally collapsing primordial clouds are very massive $\sim 1000 M_{\odot}$, since they have to be more massive than the Jean's mass, which is proportional to $T^{3 / 2}$. In addition, formation of such a massive prestellar core leads to huge mass accretion rate onto the protostar in the later mass accretion phase(e.g. Omukai \& Nishi 1998; Bromm et al. 1999; Abel et al. 2000; Nakamura \& Umemura 2001; Abel et al. 2002; Yoshida et al. 2006). Following these theoretical evidences, most of the first stars were once expected to be very massive $\left(\gtrsim 100 M_{\odot}\right)$.

On the other hand, the studies on the mass accretion phase have advanced recently, which revealed that the first star formation process seems to be more complicated than expected before(Turk et al. 2009; Clark et al. 2011a,b; Smith et al. 2011; Greif et al. 2011, 2012). They found that a heavy disk formed around the primary protostar, because of the angular momentum of the prestellar core, gained by the tidal interactions with other cosmological overdense regions. The heavy disk fragments into small pieces since it is gravitationally un-

\footnotetext{
${ }^{1}$ susa@konan-u.ac.jp
}

stable. As a result, a "star cluster" could be formed instead of a single very massive star.

These results seem to be robust until the primary protostar grows to $\gtrsim 20 M_{\odot}$. After the mass of the protostar exceeds $\sim 20 M_{\odot}$, significant ultraviolet radiation flux will be emitted from the protostar Omukai \& Palla 2003; Hosokawa \& Omukai 2009; Hosokawa et al. 2011). Thus, the ultraviolet radiation from the protostar significantly affects the later evolution of the system.

Hosokawa et al. (2011) have addressed this feedback effect directly by two dimensional radiation hydrodynamics simulations. They found that the accretion disk around the primary protostar is photoevaporated due to the radiative feedback, followed by the rapid decline of the mass accretion rate onto the protostar. The final mass of the protostar in their simulation is $43 M_{\odot}$.

Stacy et al. (2012) also tried to assess the final mass of the first stars in their three dimensional cosmological calculations including the fragmentation of the disk as well. They also found that the ultraviolet radiative feedback strongly suppress the mass accretion onto the protostars. However, the integrated physical time is $\sim 5000 \mathrm{yrs}$, which is too short to predict the final mass of the first stars, since it will take $\sim 0.1 \mathrm{Myrs}$ until the protostar settles onto the main sequence(e.g. Hosokawa \& Omukai 2009).

In this paper, we report the results of three dimensional radiation hydrodynamics simulations on the formation of first stars that follow the evolution of the system for 0.1 Myrs after the formation of the primary protostar. We take into consideration the three dimensional effects, as well as the radiative feedback from the protostars.

\section{NUMERICAL SIMULATION}

We study the formation of first stars by radiation hydrodynamics simulations. We employ the Bonner-Ebert sphere of $n_{\mathrm{H}}=10^{4} \mathrm{~cm}^{-3}, T=200 \mathrm{~K}$ as the initial condition of the simulation. This initial condition is motivated by the cosmological simulations Abel et al. 2002; Yoshida et al. 2003) in which such clouds are found in minihalos of mass $10^{5}-10^{6} M_{\odot}$, at the "loitering" 
of a protostar is obtained based on the calculation by Hosokawa \& Omukai (2009). They have calculated the evolution of protostars with given (fixed) mass accretion rates. On the other hand, we obtain the protostellar masses $(M)$ and the mass accretion rates $(\dot{M})$ self consistently from the hydrodynamics simulation. Then we can assess the luminosities and effective temperatures by interpolating the data by Hosokawa \& Omukai (2009) at every time step. These luminosities and temperatures are used to give the luminosities and black body spectra of the protostars. Hence, the protostellar evolution model is self consistently fed to radiation hydrodynamics calculations.

\section{RESULTS}

We perform radiative hydrodynamics simulations of the first star formation with radiative feedback. We also perform a run with no feedback for comparison.

\subsection{Fragmentation of the disk around the primary protostar}

We start the simulation from the rigidly rotating Bonner-Ebert sphere around the loitering phase. The cloud starts to collapse in run-away fashion, i.e. the central density keeps growing while the outer part of the cloud is left in the envelope. As a result, density profile of $\propto r^{-2.2}$ is built up during the run-away phase (Yoshida et al. 2006).

Eventually, the central density exceeds $n_{\text {sink }}$, and a sink particle is formed at the center of the cloud. The surrounding gas starts to accrete onto the sink particle subsequently. Since the gas has a significant amount of specific angular momentum, the accreting gas forms an accretion disk around the sink particle. The amount of specific angular momentum in the run-away phase is close to that of the similarity solution, which is approximately 0.5 times the value of Kepler rotation at the Jeans radius, i.e. the core radius (Yoshida et al. 2006). Thus, the radius of the disk is 0.25 times smaller than their original radius at the run-away phase, since the centrifugal force is proportional to the square of the specific angular momentum.

After the formation of the small gas disk around the first sink, the gas keeps accreting onto the disk. As a result, the mass and the radius of the disk increase. At the same time, the temperature of the disk decreases by the radiative cooling. The left column of Fig 1 shows the face-on views of the disk column density at three epochs corresponding to $320 \mathrm{yrs}, 620 \mathrm{yrs}$ and $860 \mathrm{yrs}$ after the formation of the first sink. The red crosses denote the position of sink particles.

In the early epoch of the accretion phase, a smooth disk forms around the sink particle (top panel), followed by the formation of spiral arms (middle panel) and the fragmentation of the arms (bottom panel). We can also find a few high column density peaks in the bottom panel. In fact, next sink particles are born from these peaks within a few hundred years.

of protostellar model in which steady accretion is assumed(e.g. Stahler et al. [1986). More violent/clumpy mass accretion could remain the effective temperature of protostar colder(Smith et al. 2012) to larger masses.
The right column of Fig 1 shows the color contour of Toomre's $Q$-parameter, which is given as

$$
Q \equiv c_{\mathrm{s}} \Omega_{\mathrm{orb}} /(\pi G \sigma)
$$

in case we assume Keplerian motion. Here $c_{\mathrm{s}}$ denotes the sound velocity of gas, $\Omega_{\mathrm{orb}}$ is the angular velocity around the central sink particle and $\sigma$ is the column density of the disk. In the case that the $Q$-parameter is less than unity, a smooth disk with density perturbations becomes gravitationally unstable. In fact, the $Q$-parameter of the disk at the early phase (top) is already less than unity, so the disk is unstable (middle and bottom).

The time scale of disk instability is given by the linear perturbation theory (Toomre 1964), which is given as $\Omega_{\text {orb }}^{-1}\left(Q^{-2}-1\right)^{-1 / 2}$. The typical value of $Q$-parameter in the disk at early phase (top) is $\sim 0.5$, and the angular velocity is $\Omega_{\text {orb }} \simeq 10^{-9} \mathrm{~s}^{-1}$. Thus the perturbation growth time scale is $\sim 20 \mathrm{yrs}$, which is comparable to the time scale of the generation of the spiral structure. Thus, spiral structures seems to develop due to the gravitational instability, which could be understood as Toomre's criteria. On the other hand, it takes several hundred years for another sink to be born (bottom), and seemingly via the fragmentation of the spiral arms. Therefore, formation of the sink particles in the disk cannot be understood solely by the simple $Q$-parameter argument above, but requires non-linear calculations.

\subsection{Effects of radiative feedback}

Fig 2 2 illustrates the evolution of the edge-on view of the central $10^{4} \mathrm{AU}(0.05 \mathrm{pc})$ in radius. The color shows the fraction of $\mathrm{H}_{2}$ molecules, and the transparency denotes the gas density. Small spheres are the position of sink particles. Initially, $\mathrm{H}_{2}$ fraction is quite high $\left(y_{\mathrm{H}_{2}} \sim 10^{-2}\right.$, upper left panel). Eventually, the polar region is photodissociated as the sink particles grow (top right). Some $\mathrm{H}_{2}$ rich regions remain along the equatorial plane due to the self-shielding (bottom left), but finally they disappear after 0.1 Myrs (bottom right).

Fig 3 illustrates the evolution of the system on densitytemperature plane. Color map shows the frequency distribution of SPH particles on the plane. Four panels show the snapshots at $t=-10 \mathrm{yr}, 2450 \mathrm{yr}, 5510 \mathrm{yr}, 100250 \mathrm{yr}$, respectively. The distribution of SPH particles on the top left panel is similar to the well known curve of the evolution of collapsing primordial gas in run-away phase (e.g. Palla et al. 1983), since it corresponds to the epoch just before the first sink formation. After the first sink formation, dense gas $\left(\gtrsim 10^{10} \mathrm{~cm}^{-3}\right)$ is split to high temperature gas $(\lesssim 7000 \mathrm{~K})$ and low temperature gas $(\lesssim 1000 \mathrm{~K}$, top right panel). The former corresponds to the radiatively heated gas and the shock heated gas, while the latter is the self-shielded cold gas orbiting around the sink particles. Then, the high temperature gas in the high density region expands due to the increased thermal pressure, generating a shock propagating to low density region (bottom left panel). In fact, we also have seen the shock wave in the bottom left panel of Fig,2, marked by white dashed curve. Finally, the dense cold gas disappears (bottom right), which means that star formation no longer proceeds.

The solid line in Fig 4 shows the time evolution of the total mass in the sink particles in the feedback run, 
Column density $\left[\mathrm{g} / \mathrm{cm}^{-2}\right]$
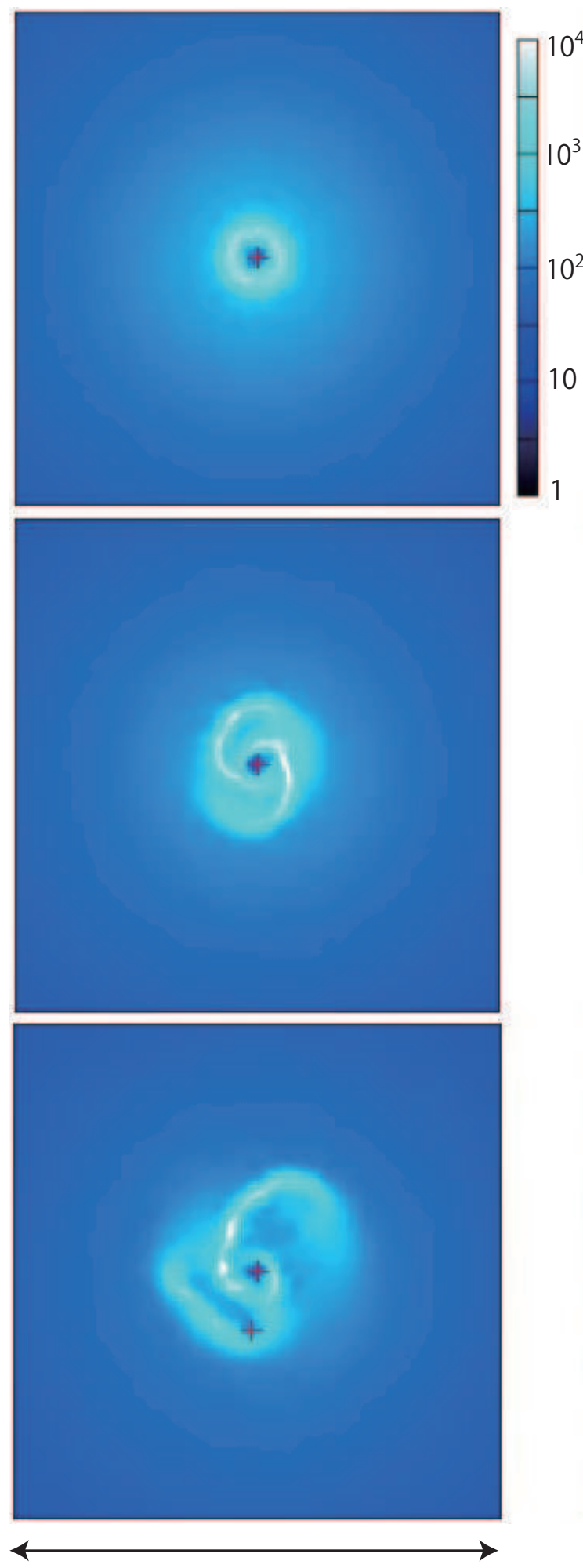

1200AU
Q-parameter
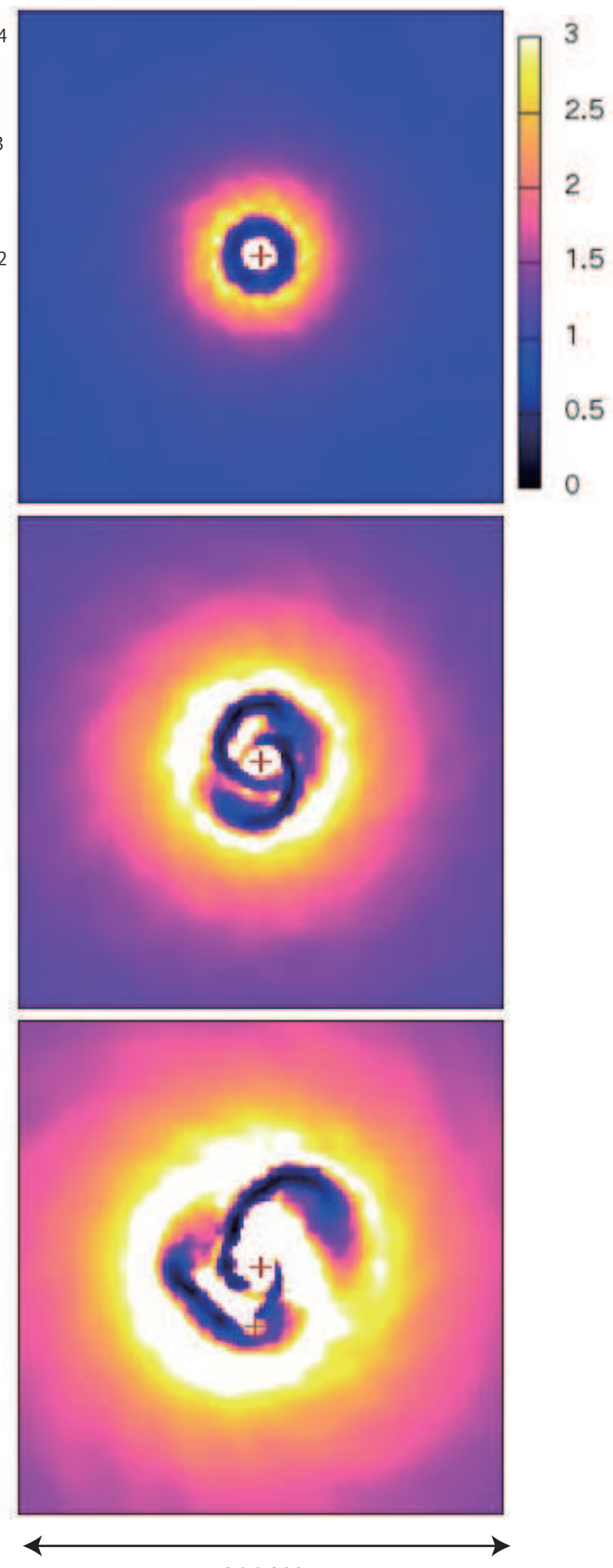

1200AU

Fig. 1. - Face-on view at the beginning of the accretion phase. Left column: Three snapshots (320yr, 620yr, 860yr from top to bottom) of color contour of the gas column density. Right column: Toomre's Q-parameter at the same moment. 


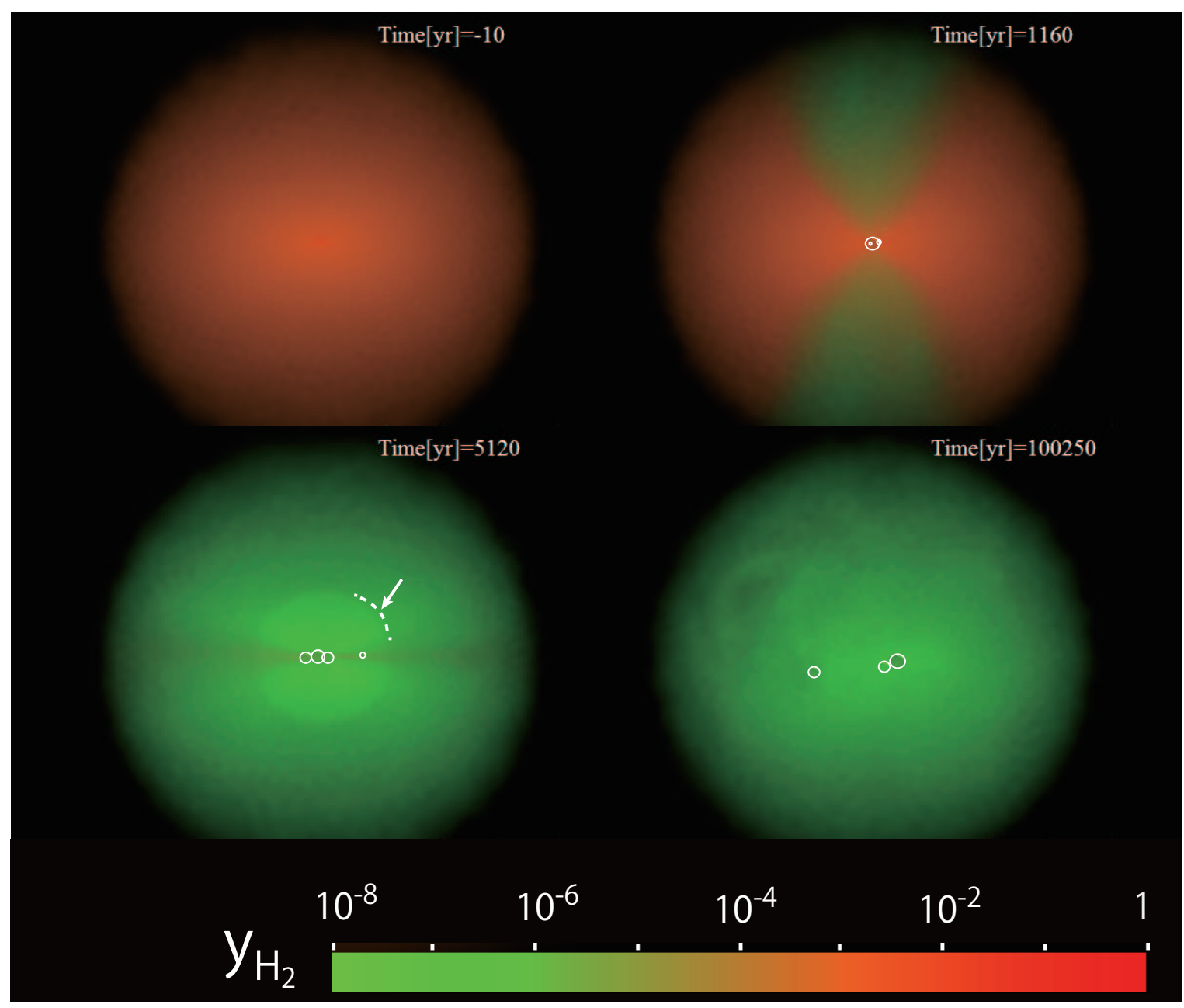

FIG. 2. - Edge-on views of gas distribution inside $r<10^{4} \mathrm{AU}(0.05 \mathrm{pc})$ at four snapshots. Top row: from left to right, $t=-10 \mathrm{yr}, 1160 \mathrm{yr}$. Bottom row: $t=5120 \mathrm{yr}, 100250 \mathrm{yr}$. $t$ represents the time after the first sink formation. Color shows the $\mathrm{H}_{2}$ fraction, and the small spheres with white rim represent the positions of sink particles. White arrow and dashed curve in the bottom left panel denotes the position of the shock front. 


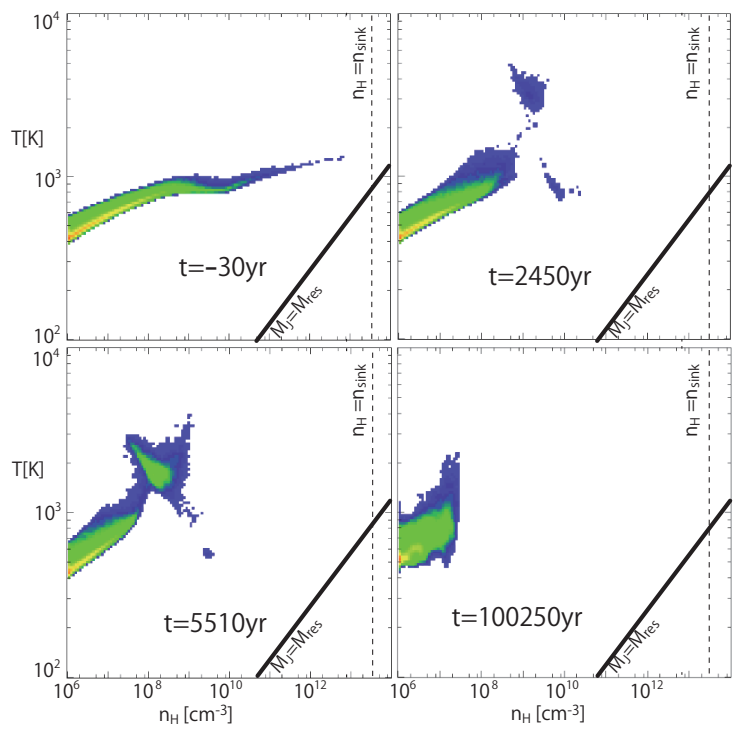

FIG. 3.- Four snapshots on the density-temperature plane. Color represents the number of SPH particles drop on the logarithmic bin on the plane. Thick solid lines show the resolution limit of this simulation, while dashed lines represent the number density above which the SPH particles are converted to sink particles.

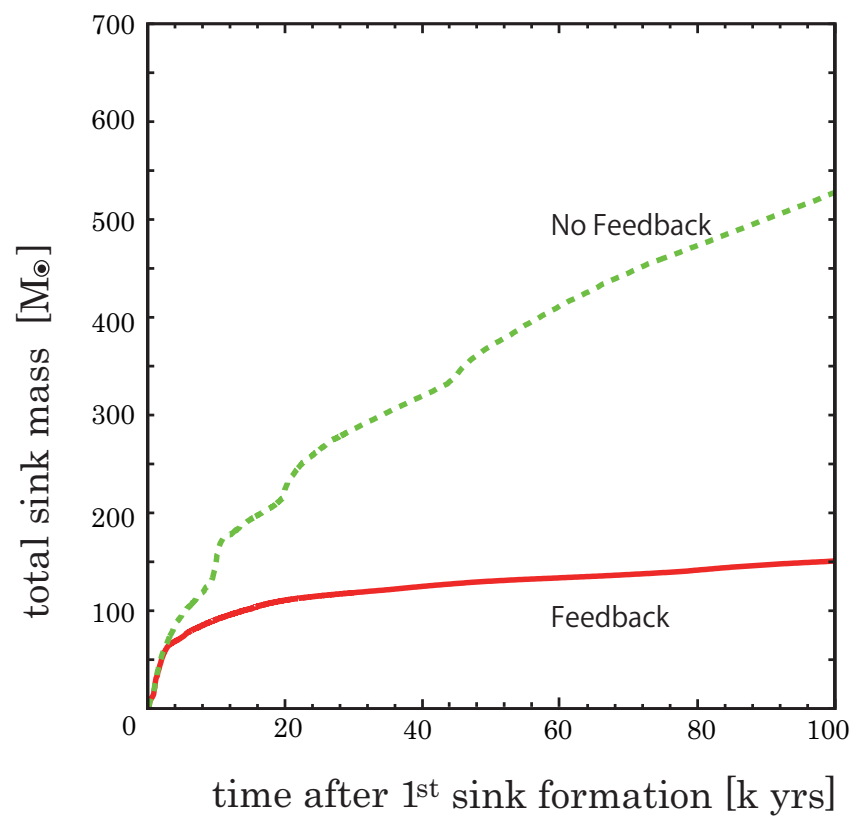

FIG. 4.- Evolution of the total mass incorporated in sink particles. The solid line corresponds to the case with feedback, while the dashed line represents the case without feedback.

whereas the dashed line represents that without radiative feedback effects. It is clear that the mass accretion onto the sink particles is highly suppressed by the radiative feedback. The total sink mass of the run with feedback at the end of the simulation $(\sim 0.1$ Myr $)$ is less than a third of that without feedback. We also find that the mass accretion rate in feedback run is smoother than that of no feedback run. This is because the gas in the

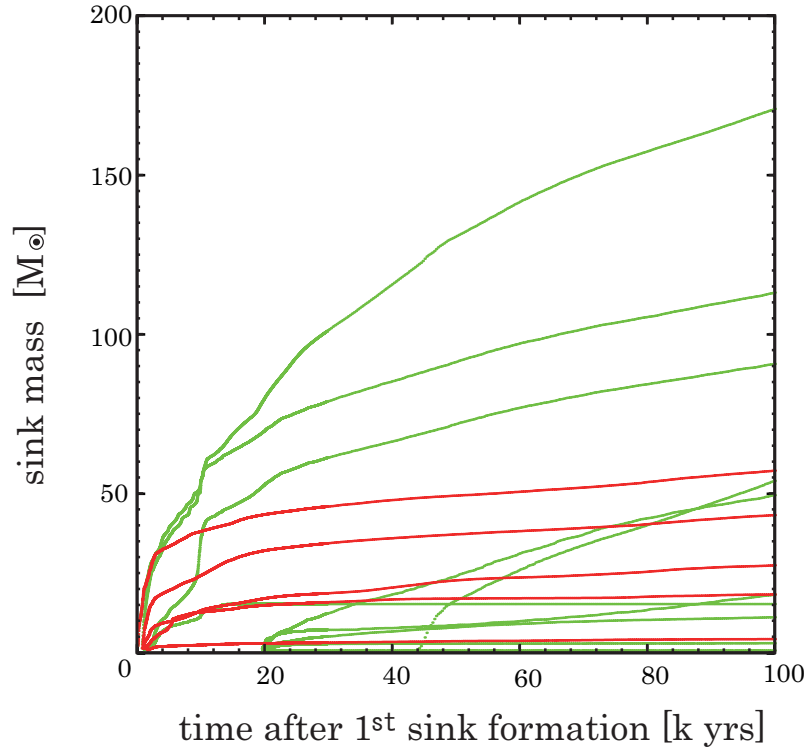

FIG. 5.- The mass of individual sink particle is plotted as a function of time after the first sink formation. Red curves for the runs with feedback, while the green curves for the case without feedback.

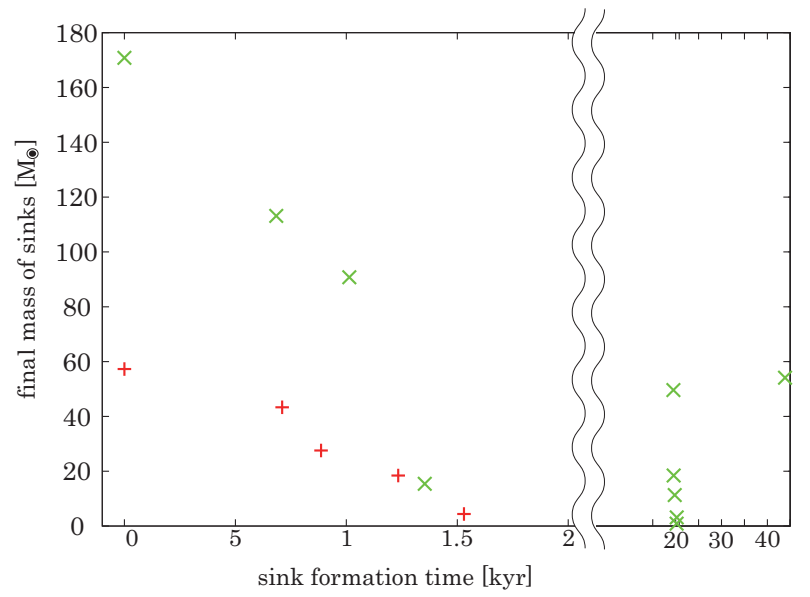

FIG. 6.- Formation time of individual sink particle v.s. final mass. Red crosses : feedback run, Green vertices: no feedback run.

latter case is more clumpy than that of the former, due to the absence of additional heating processes provided by the ultraviolet radiation from the protostar. Fig 5 illustrates the time evolution of the mass of each sink particle. The red curves represent those in the run with feedback, while the green curves are those results without feedback. In the feedback run, we have one star more massive than $50 M_{\odot}$ at $0.1 \mathrm{Myr}\left(\sim 57 M_{\odot}\right)$, whereas we have three stars in the range of $50-200 M_{\odot}$ in the no feedback run.

The minimal sink mass in the feedback run is $4.4 M_{\odot}$, and the total number of the sink particles is 5 . On the other hand, in the no feedback run, the minimal sink mass is $0.84 M_{\odot}$, and the total number of sink particles is 10. It is also worth noting that fragmentation of the disk in the no feedback case continues until much later time $\left(\sim 4 \times 10^{4} \mathrm{yr}\right)$ than that in the feedback run $(\lesssim 1500 \mathrm{yr})$, because the molecular rich disk is not destroyed until much later phase in the no feedback run. The difference in the number of sink particles and the minimal mass 


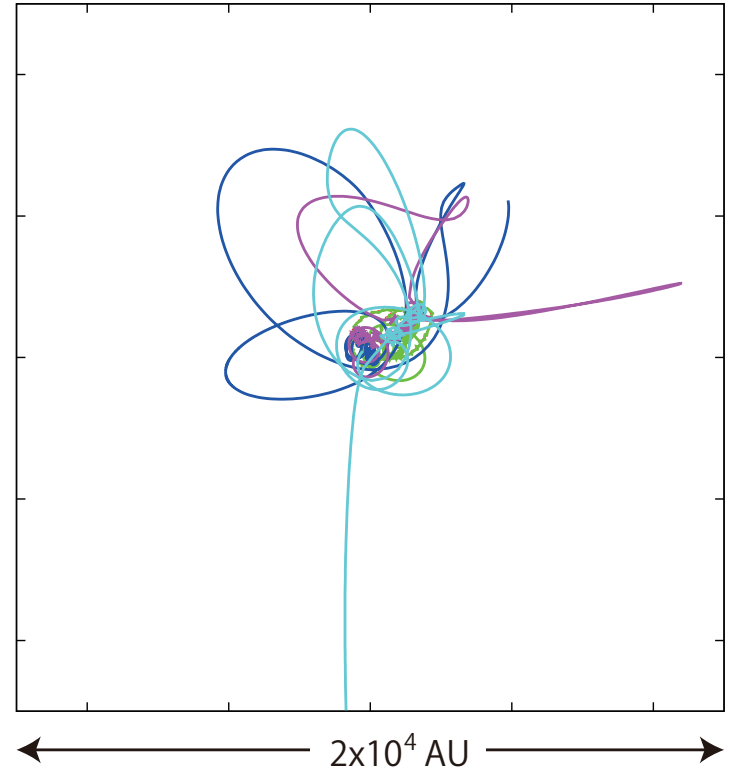

FIG. 7.- The trajectories of sink particles in edge-on view.

might come from such effect. However, it is premature to draw definitive conclusion on this issue, since our results are based upon only a single realization.

Fig 6 plots the sink formation time and the final mass (at $10^{5}$ yr after 1 st sink) for all sink particles. Red crosses denote the sinks found in feedback run, while the green vertices are in the no feedback run. Basically, earlier formation leads to more massive sinks because massive sinks gather gas more efficiently than less massive ones. This trend is clear in the feedback run, while some different behaviours are found in no feedback case. In the latter case, the gas distribution is more clumpy, which allows the sink formation at late epochs such as $20 \mathrm{kyr}$ or 40kyr after the first sink formation, as mentioned in the previous paragraph. In such cases, the growth of sinks is not affected by the sinks formed at the first episode, because it proceeds at relatively spatially distant position from the first sinks.

\subsection{Ejections}

In the simulation with feedback, we find a sink particle is kicked away from the central dense region via the gravitational N-body interaction, so-called "slingshot" mechanism. Fig 7 shows the trajectories of all the sink particles within $\left(2 \times 10^{4} \mathrm{AU}\right)^{3}$ box at the central region. It is clear that one escaping sink goes away from the central region (to the bottom of the panel), whereas the others remain within the box. Such a phenomenon has already been reported by other groups without radiative feedback effects(e.g. Smith et al. 2011). Thus, we confirm the theoretical existence of such escapers also in our numerical model with radiative feedback. The velocity of this escaping sink is $\sim 4 \mathrm{~km} / \mathrm{s}$ at $0.1 \mathrm{pc}$ distant from the center of the cloud, which is marginal to escape from the host minihalo of $10^{6} M_{\odot}$.

The mass accretion onto this escaping particle almost stops after the ejection. Consequently, the mass of the sink is $4.4 M_{\odot}$, that are much smaller than the "conventional" first stars of mass $\gtrsim 100 M_{\odot}$. It is also worth noting that the orbit of the other sinks are excited by the N-body interaction with each other, although they are not ejected. Thus, some of the sinks going through relatively low density regions, which results in low mass accretion rate onto these sinks.

We also remark that a star less massive than $0.8 M_{\odot}$ is found in our higher resolution run $\left(M_{\text {res }}=0.1 M_{\odot}\right)$ with feedback, although the integrated physical time is $\sim 2 \times 10^{4}$ yrs (Umemura et al. 2012). Considering the fact that the mass resolution and the accretion radius of the present simulations are $\sim 0.5 M_{\odot}$ and $30 \mathrm{AU}$, and other higher resolution studies with/without feedback effects report the ejection of even lower mass stars (Umemura et al. 2012; Clark et al. 2011a,b; Smith et al. 2011; Greif et al. 2011, 2012), we presume that low mass stars less massive than $0.8 M_{\odot}$ are born among the first stars, and survive through the entire history of the universe.

\section{DISCUSSIONS \& CONCLUSION}

In the presence of radiative feedback, the gas in the neighborhood of protostars is heated up significantly. This heating process occurs mainly through photodissociation of $\mathrm{H}_{2}$ molecules: Formation of $\mathrm{H}_{2}$ molecules works as a heating process of the gas, since formation process such as $3 \mathrm{H} \rightarrow \mathrm{H}_{2}+\mathrm{H}$ or $\mathrm{H}^{-}+\mathrm{H} \rightarrow \mathrm{H}_{2}+\mathrm{e}^{-}$releases the latent heat. In the absence of radiative dissociation process, collisional dissociation processes absorb thermal energy, which balance with the formation heating. Thus, the net increase of $\mathrm{H}_{2}$ molecules causes effective heating of the gas, while the net decrease of $\mathrm{H}_{2}$ results in cooling. On the other hand, in the presence of strong photodissociative radiation, it overwhelms other collisional dissociation processes. The photodissociation process do not absorb thermal energy, since the energy required to dissociate $\mathrm{H}_{2}$ molecules is supplied by the radiation. Therefore, $\mathrm{H}_{2}$ dissociation is no longer a cooling process. As a result, $\mathrm{H}_{2}$ formation heating proceeds without hindrance in the absence of the counter process. In the present simulation, strong LW radiation field from the protostar is the source of this heating process in dense regions. Consequently, this heating process drive the shock wave found in Fig,2, as well as the termination of mass accretion onto the protostars.

In fact, Fig 8 illustrates the ratio between the $\mathrm{H}_{2}$ formation heating rate and the adiabatic heating rate as functions of gas temperature and density at $t=2450 \mathrm{yr}$. It is clear that $\mathrm{H}_{2}$ formation heating is the dominant heating process at high densities $\left(n_{\mathrm{H}} \gtrsim 10^{10} \mathrm{~cm}^{-3}\right.$, right panel). The temperature of these chemo-heated high density regions is mainly around $1000 \mathrm{~K}$, and the chemical heating is also important for high temperature region at $3000 \mathrm{~K} \lesssim T \lesssim 7000 \mathrm{~K}$ (left panel). It is also clear that chemical heating rate is not negligible compared to the adiabatic heating rate even at lower densities $\left(\sim 10^{6} \mathrm{~cm}^{-3}\right)$ and lower temperatures $(T \sim 300 \mathrm{~K})$, which corresponds to the dissociated polar regions. Thus, chemical heating of $\mathrm{H}_{2}$ formation play important roles on the dynamical evolution of this system.

Heating through photoionization is also important. In fact, similar calculation in 2D by Hosokawa et al. (2011), photoionization is the dominant heating process. They found the breakout of ionization fronts into the polar region, and the gas is highly ionized and heated up to 

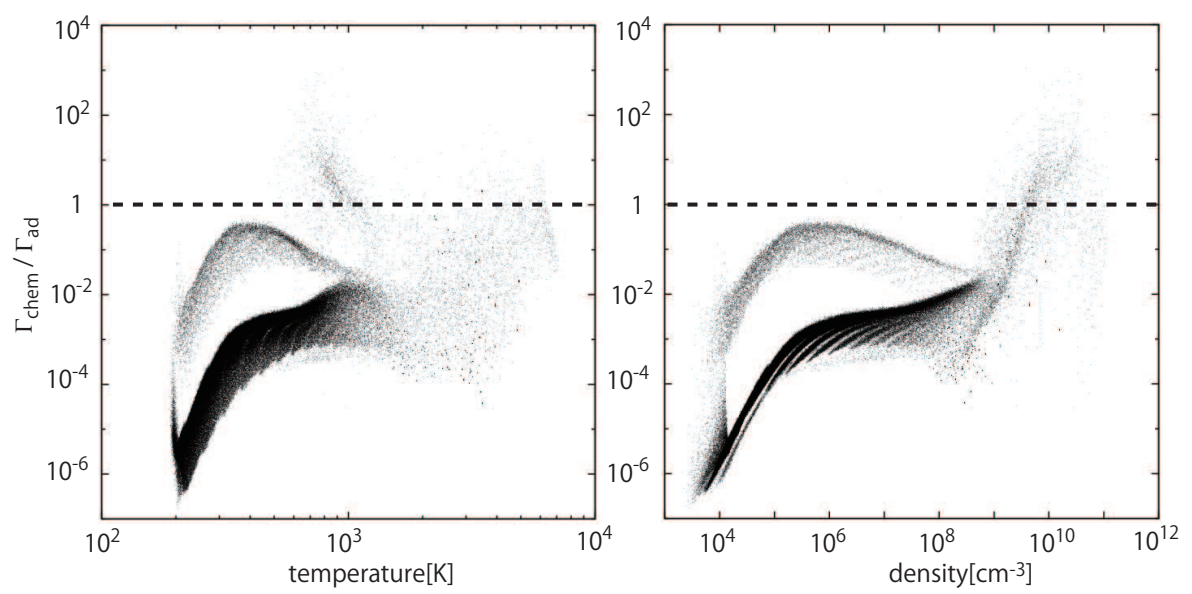

FIG. 8. - The ratio between the chemical heating rate and the adiabatic heating rate is plotted as functions of temperature (left panel) and density (right panel) at $t=2450 \mathrm{yr}$.

$>10^{4} \mathrm{~K}$. Finally, the disk is photoevaporated mainly due to the photoheating process through ionization. In the present calculation, however, ionization is not the dominant heating process. The reason simply comes from the fact that the spatial resolution of the present simulation is not enough to resolve the "initial" Strömgren sphere(Spitzer 1978). In Fig,9, we plot the ratio between the Strömgren radius and the SPH spatial resolution at given densities. Here the mass accretion rate is assumed to be $10^{-4} M_{\odot} / y r$, which is a typical value in present calculation at $10 \mathrm{kyrs}-100 \mathrm{kyrs}$ after the first sink formation. It is clear that if the gas densities in the neighbor of the protostar is larger than $\sim 10^{6} \mathrm{~cm}^{-3}$, the initial Strömgren radius cannot be resolved by the resolution of the present simulation. In the present simulation, gas particles in the neighbor of the source stars are always denser than $10^{7} \mathrm{~cm}^{-3}$, which is too high to capture the propagation of ionization fronts. Hence the SPH particles only in the very vicinity of the source stars are heated by ionization "mildly", $T \lesssim 10^{4} \mathrm{~K}$, which cannot halt the mass accretion onto the protostar and cannot cause the breakout of D-type ionization front which is found in Hosokawa et al. (2011). In other words, this radiative feedback due to photoheating effect is heavily underestimated in the present simulation.

As noticed in section 2, the algorithm of mass accretion onto sink particles overestimates the mass accretion rate. Combined with the fact that feedback is underestimated, the mass accretion rate obtained in the present numerical experiment is overestimated at any hand. Accordingly, the final mass of the sinks should be regarded as an upper limit of the actual mass of first stars. If we could perform simulations at higher resolution with more realistic mass accretion conditions, the final mass could be smaller than $\sim 50 M_{\odot}$. We also should keep in mind that this result comes from a numerical experiment of one realization. Thus, this upper limit should be regarded as a guide, since a slightly different initial condition could cause different result because of the chaotic nature of the system. On the other hand, at least one of the protostars cannot be less massive than $M \sim 20 M_{\odot}$, since the radiative feedback becomes prominent only for $M \gtrsim 20 M_{\odot}$. Thus, the mass of the primary star among the first stars formed in a single mini-halo will fall in the range of $20 M_{\odot}$-several $\times 10 M_{\odot}$.

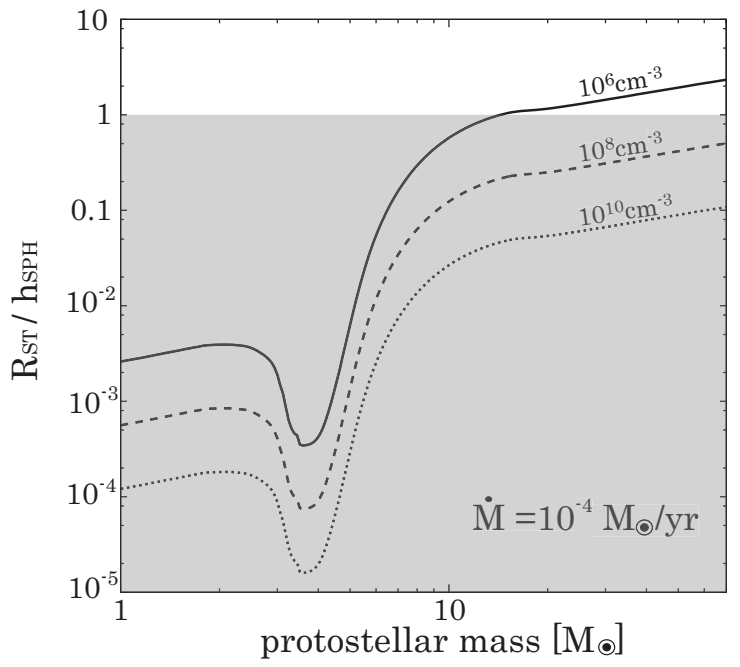

FIG. 9.- The ratio between the Strömgren radius $R_{\mathrm{ST}}$ and the SPH spatial resolution $h$ at given densities. Three curves correspond to $n_{\mathrm{H}}=10^{10} \mathrm{~cm}^{-} 3,10^{8} \mathrm{~cm}^{-} 3,10^{6} \mathrm{~cm}^{-} 3 . R_{\mathrm{ST}}$ is not resolved in the shaded region.

We also remark that the evidences of $15-$ $50 M_{\odot}$ population III stars have known for several years(Beers \& Christlieb 2005; Frebel et al. 2005; Cavrel et al. 2004; Iwamoto et al. 2005; Lai et al. 2008; Joggerst et al. 2010; Caffau et al. 2012), which are consistent with the present results. In addition, however, present results do not exclude the presence of very massive stars of $>130 M_{\odot}$ which result in pair instability supernovae, since present simulation is a result of one realization. Search for the evidences of strong "odd-even" effects known as the mark of pair instability supernovae, in dumped Lyman- $\alpha$ systems (Cooke et al. 2011) or metal poor stars (Ren et al. 2012) could give the constraint on this type theoretical experiments.

The initial condition of the present experiment is a rigidly rotating Bonner-Ebert sphere. Although its angular momentum distribution just before the sink formation is close to that of cosmological simulations, it is not fully cosmological. In cosmological simulations, the direction of angular momentum of the disk around the primary protostar changes depending on the stages, since the gas motion is more turbulent. In addition, we need more numerical experiments starting from various initial 
conditions, in order to obtain statistical quantities such as the initial mass function (IMF) of first stars. Thus it is important to perform fully cosmological simulations of this sort, which is left for future works.

In this paper, we investigated the suppression of mass accretion onto population III proto-stars by the radiative feedback from themselves. We performed numerical experiment of the formation of first stars using three dimensional radiative hydrodynamics code RSPH combined with sink particle technique. Consequently, we find that the mass accretion is suppressed significantly and the final outcome is a multiple stellar system consisting of five stars of $1-60 M_{\odot}$. The fact that low mass stars are found in this work infer the possible existence of first stars in the local universe, although the mass of the formed stars in our simulation is still larger than 0.8 $M_{\odot}$.

We thank anonymous referee for his/her careful reading and constructive comments. We also thank $\mathrm{T}$. Hosokawa for providing the data of protostars and K. Omukai for careful reading of the manuscript. This work was supported by Ministry of Education, Science, Sports and Culture, Grant-in-Aid for Scientific Research (C), 22540295 .

\section{REFERENCES}

Abel, T., Bryan, G. L., \& Norman, M. L. 2000, ApJ, 540, 39 Abel, T., Bryan, G. L., \& Norman, M. L. 2002, Science, 295, 93 Bate, M. R., Bonnell, I. A., \& Price, N. M. 1995, MNRAS, 277, 362

Bate, M. R., \& Burkert, A. 1997, MNRAS, 288, 1060

Beers, T. C., \& Christlieb, N. 2005, ARA\&A, 43, 531

Bromm, V., Coppi, P. S., \& Larson, R. B. 1999, ApJ, 527, L5

Bromm, V., Coppi, P. S., \& Larson, R. B. 2002, ApJ, 564, 23

Caffau, E., Bonifacio, P., François, P., et al. 2012, A\&A, 542, A51

Cayrel, R., Depagne, E., Spite, M., et al. 2004, A\&A, 416, 1117

Clark, P. C., Glover, S. C. O., Smith, R. J., et al. 2011, Science, 331, 1040

Clark, P. C., Glover, S. C. O., Klessen, R. S., \& Bromm, V. 2011, ApJ, 727, 110

Cooke, R., Pettini, M., Steidel, C. C., Rudie, G. C., \& Jorgenson, R. A. 2011, MNRAS, 412, 1047

Frebel, A., Aoki, W., Christlieb, N., et al. 2005, Nature, 434, 871

Fuller, T. M., \& Couchman, H. M. P. 2000, ApJ, 544, 6

Galli, D., \& Palla, F. 1998, A\&A, 335, 403

Greif, T. H., Bromm, V., Clark, P. C., et al. 2012, MNRAS, 424, 399

Greif, T. H., Springel, V., White, S. D. M., et al. 2011, ApJ, 737, 75

Haiman, Z., Thoul, A. A., \& Loeb, A. 1996, ApJ, 464, 523

Hosokawa, T., \& Omukai, K. 2009, ApJ, 691, 823

Hosokawa, T., Omukai, K., Yoshida, N., \& Yorke, H. W. 2011, Science, 334, 1250

Iwamoto, N., Umeda, H., Tominaga, N., Nomoto, K., \& Maeda, K. 2005, Science, 309, 451

Joggerst, C. C., Almgren, A., Bell, J., et al. 2010, ApJ, 709, 11

Kepner, J. V., Babul, A., \& Spergel, D. N. 1997, ApJ, 487, 61

Lai, D. K., Bolte, M., Johnson, J. A., et al. 2008, ApJ, 681, 1524

Martel, H., Evans, N. J., II, \& Shapiro, P. R. 2006, ApJS, 163, 122

Nakamura, F., \& Umemura, M. 2001, ApJ, 548, 19
Nishi, R., \& Susa, H. 1999, ApJ, 523, L103

Omukai, K. 2000, ApJ, 534, 809

Omukai, K., \& Nishi, R. 1998, ApJ, 508, 141

Omukai, K., \& Palla, F. 2003, ApJ, 589, 677

Palla, F., Salpeter, E. E., \& Stahler, S. W. 1983, ApJ, 271, 632

Ren, J., Christlieb, N., \& Zhao, G. 2012, Research in Astronomy and Astrophysics, 12, 1637

Ripamonti, E., \& Abel, T. 2004, MNRAS, 348, 1019

Shapiro, P. R., \& Kang, H. 1987, ApJ, 318, 32

Smith, R. J., Glover, S. C. O., Clark, P. C., Greif, T., \& Klessen, R. S. 2011, MNRAS, 414, 3633

Smith, R. J., Hosokawa, T., Omukai, K., Glover, S. C. O., \&

Klessen, R. S. 2012, MNRAS, 424, 457

Spitzer, L. 1978, New York Wiley-Interscience, 1978. 333 p.249

Stacy, A., Greif, T. H., \& Bromm, V. 2012, MNRAS, 422, 290

Stahler, S. W., Palla, F., \& Salpeter, E. E. 1986, ApJ, 302, 590

Stancil, P. C. 1994, ApJ, 430, 360

Susa, H. 2006, PASJ, 58, 445

Susa, H., \& Umemura, M. 2004, ApJ, 600, 1

Tegmark, M., Silk, J., Rees, M. J., et al. 1997, ApJ, 474, 1

Toomre, A. 1964, ApJ, 139, 1217

Turk, M. J., Abel, T., \& O'Shea, B. 2009, Science, 325, 601

Umemura, M., Susa, H., Hasegawa, K., Suwa, T., \& Semelin, B. 2012, Prog. Theor. Exp. Phys, 01A306

Wolcott-Green, J., Haiman, Z., \& Bryan, G. L. 2011, MNRAS, 418,838

Yoshida, N., Abel, T., Hernquist, L. \& Sugiyama, N., 2003, ApJ, 592,645

Yoshida, N., Omukai, K., Hernquist, L., \& Abel, T. 2006, ApJ, 652,6

Yoshida, N., Omukai, K., \& Hernquist, L. 2008, Science, 321, 669 